\title{
NexGenEx-Tom: a gene expression platform to investigate the functionalities of the tomato genome
}

\author{
Hamed Bostan and Maria Luisa Chiusano*
}

\begin{abstract}
Background: Next Generation Sequencing technologies (NGS) unexpectedly pushed forward the capability of solving genome organization and of widely depicting gene expression. However, although the flourishing of tools to process the NGS data, versatile and user-friendly computational environments for integrative and comparative analyses of the results from the increasing amount of collections are still required.

The gene expression of tomato tissues has been widely investigated in the years, thanks to both EST sequencing and different microarray platforms. However, the resulting collections are heterogeneous in terms of experimental approaches, genotypes and conditions, making the data far from representing a gene expression atlas for the species. Therefore, the recent release of NGS transcriptome collections from several tissues and stages from physiological conditions for specific tomato genotypes provides a relevant resource to be appropriately exploited to address key questions on gene expression patterns, such as those related to fruit ripening and development in tomato. The organization of the results from the processed collections in web accessible environments, enriched with tools for their exploration, may represent a precious opportunity for the scientific research in tomato and a reference example for similar efforts.

Description: Here we present the architecture and the facilities of NexGenEx-, a web based platform that offers processed NGS transcriptome collections and enables immediate analyses of the results. The platform allows gene expression investigations, profiling and comparisons, and exploits different resources.

Specifically, we present here the platform partition NexGenEx-Tom, dedicated to the organization of results from tomato NGS based transcriptomes.

Conclusion: In the current version, NexGenEx-Tom includes processed and normalized NGS expression data from three collections covering several tissue/stages from different genotypes. Beyond providing a user-friendly interface, the platform was designed with the aim to easily be expanded to include other NGS based transcriptome collections. It can also integrate different genome releases, possibly from different cultivars or genotypes, but even from different species. The platform is proposed as an example effort in tomato, and is described as a profitable approach for the exploitation of these challenging and precious datasets.
\end{abstract}

Keywords: Tomato expression database, NGS data analysis, Expression Atlas, Gene expression profiling, Gene expression comparison

\footnotetext{
* Correspondence: chiusano@unina.it

Department of Agricultural Sciences, University of Naples "Federico II", via Università 100, 80055 Portici, Italy
} 


\section{Background}

Nowadays, the development of high throughput methodologies and advanced genomics are leveraging the genomewide characterization of many different species [1]. Next Generation sequencing (NGS) technologies are strongly supporting this progress, being extensively exploited for both genome sequencing and gene expression investigations. These novel approaches play a key and unexpected role for their impressive capability to offer an in depth scanning of the transcriptome level of the cell functionality, providing a consistent contribution to the exploration of the genome expression, its control and related regulatory mechanisms. Moreover, NGS based transcriptome collections provide extended knowledge of gene structure and of expression of alternatively spliced gene models. Nonetheless, though these technologies are expanding, also because of their cost affordability [2], even to not specialized research centers, the large amount of data produced makes straightforward exploitations still a challenge for the scientific community.

Reference platforms such as Sequence Read Archive $(S R A)$ [3] and Gene Expression Omnibus (GEO) [4] have been established to gather the NGS primary data collections, i.e. those directly provided by the sequencing centers in raw formats.

A significant number of tools and software have been released to pre-process (cleaning, trimming and quality checking [5,6]), process (de novo assembling $[7,8]$, mapping to the genome sequence $[9,10])$, and analyze $[11,12]$ the NGS data. Platforms including tools and pipelines provided with appropriate interfaces are flourishing to fulfill the need to support the transferring from raw data to accessible and meaningful biological information. Some interesting examples comes from the Galaxy project [13], which is a comprehensive open source toolbox meant to offer an interactive web based platform for running and tracing the manifold bioinformatics analyses useful for genomics, also permitting workflows on varied data types, NGS libraries included. Interestingly, the platform evolves thanks to a collaborative community of users and developers as a shared effort in an open source framework. GenePattern 2.0 [14] provides access to a large number of tools not limited to RNA-seq analyses, also permitting the creation of multi-step pipelines, allowing the definition of appropriate workflows for reproducible analyses. NGS-Trex [15] is a web based platforms, exclusively dedicated to NGS data analysis, offering tools within a predefined workflow. InSilico $D B$ [16] is a web based data storage and processing hub, free for basic usage and under payment for expanded analyses. It offers access to large pre-processed datasets from different species, but also methods for stand-alone genome wide analyses. The CLC bio integrated platform [17] offers a wide range of software and tools for biological data analyses. Processing toolboxes and computational power for RNA-seq data management in the form of online or stand-alone tools are available for free (Sequence viewer), or under payment, if requested in the form of workbenches.

In terms of visualizing NGS based results, the Integrative Genomics Viewer (IGV) [18] and Gbrowse [19] efficiently handle large heterogeneous datasets, while providing a smooth and intuitive user access to all levels of genome resolution. Both platforms support NGS data visualization in the form of short reads and coverage plots along genomic sequences.

Some other efforts specifically aim to provide immediate tools to investigate collections of processed data, for integration and data sharing. As an instant RNASeqExpressionBrowser [20] provides query interfaces by gene identifiers (ID), by keyword or by sequence, exploiting data results uploaded in a matrix-like format. There are also several, more specific reference sites, offering tools for the visualization of the gene expression results, mainly in the form of single dimension processed collections (plain tab delimited or csv formatted files including the genes and their expression levels as raw reads count or defined by specific normalization approaches) or in the form of reads mapped on the genome and accessible by a suitable genome browser interface. As an example, the Ensembl Genome Browser [21] provides NGS data for many differ species, in the form of short read tracks mapped onto the corresponding reference genome sequence and visually accessible from the associated genome browser interface, supporting scripting and APIs for in-depth comparative analysis and data mining [22].

In Plants, several resources are dedicated to species specific collections. The TIGR Rice Annotation project [23] includes data from RNA-seq collections offers tools for gene based investigations and for genome based views of their mapped distribution.

SoyBase [24], the USDA-ARS soybean genetic database, also includes RNA-seq expression data from a collection including different libraries, offering search, comparative and clustering tools for the analyses.

The Potato Genomic Resource (Spud DB) [25] also provides potato RNA-seq data in the form of raw short read sequence files and of FPKM normalized results as defined by the Cufflinks pipeline [26] (analogous to the RPKM normalization [27]) in Excel format, as well as visualization of expression tracks by library in a potato genome browser.

The Maize gene atlas [28] also provides a consistent RNA-seq collections of 18 tissues representing five organs. This dataset, together with microarray data from 60 unique spatially and temporally separated tissues from 11 maize organs, offers a comprehensive collection for understanding gene transcription during maize development. 
An RNA-seq expression atlas [29] for common bean has also been recently released.

We here propose NexGenEx- as a bioinformatics platform designed to make pre-processed NGS based transcriptome collections available for suitable exploration of gene expression based on a reference genome sequence, when the corresponding gene annotation is available. In particular, as an example of application, we here describe the partition dedicated to tomato: NexGenEx-Tom.

Recently, the tomato genome sequencing Consortium released the preliminary version of the pseudomolecules of S. lycopersicum, the common tomato [30]. Tomato is the most extensively investigated Solanaceae species and it is a model system for fruit development. Defining its genome organization, its gene content and the related expression is relevant for addressing several open questions, such as the underlying mechanisms involved in fruit ripening and development, quality and yield. Moreover, these issues are also fundamental for comparative analyses with related genomes, such as those from wild relatives or from other Solanaceae species, like potato [31], pepper [32], Eggplant [33], or from other species with fleshy fruits, like grapevine. The 12 tomato pseudomolecules represent a first reference of the sequences of the tomato chromosomes, resulting in $\sim 760 \mathrm{Mb}$ (the expected total size is estimated to be $\sim 900 \mathrm{Mb}$ ) [30]. Specifically, the Consortium sequenced the Heinz 1706 cultivar and also produced a collection of Illumina based transcriptome sequences from the same genotype. This collection includes two biological replicates from 10 different samples, from tissues and developmental stages in physiological conditions. The aim of the effort was to enrich the release of the genome sequence with a comprehensive overview of its expression, and to release preliminary results depicting the expression patterns associated to fruit settings and development. Moreover, RNA-seq data were also provided from different samples in physiological conditions of the putative closest wild relative Solanum pimpinellifolium [30]. Recently, similar data from different stages of fruit development from the genotype Ailsa Craig have been also provided [34].

The three NGS based collections are relevant if comparatively exploited since they offer insights on gene expression from several tissue/stages from different related genotypes, but also because they offer the opportunity to extensively investigate tissue specific gene expression patterns.

Several resources offer tomato gene expression data. The Tomato Functional Genomics Database (TFGD) [35] is a website specifically aimed to provide a representative resource for gene expression collections from tomato, including data from heterogeneous platforms (ESTs, microarrays, RNA-seq). Among different RNA-seq collections, the platform also organizes results from the three collections we described, offering RPKM normalized data per library when querying for a selected collection by gene ID or keyword.

The Solanaceae Genomic Network (SGN) website [36] is offering combined results from RNA-seq from unspecified collections in the form of short reads mapped onto the genome and accessible by a genome browser interface as coverage plots.

To our knowledge, the Tomato Genomic Resources Database (TGRD) [37] is the other tomato related resource also providing the RNA-seq based expression of tomato genes in selected tissues (leaf, root, flower and fruit) from the Heinz reference collection [30].

NexGenEx-Tom is dedicated to the organization of gene expression data from tomato, currently including the three publically available tomato collections from different genotypes, representing several tissues and stages from physiological conditions. As a novelty in the framework of tomato genomics, it offers different tools to explore and mine the processed and normalized data permitting straightforward, flexible analyses, abstracting results in different user-friendly views and offering the possibility to customize the investigations on single and/or multiple genes sets defined according to suitable queries.

In the current settings, NexGenEx-Tom offers a useful resource for tomato, because it permits to friendly explore the genome functionality and to straightforward investigate NGS based gene expression patterns in physiological conditions. This environment is designed to be easily expanded with new libraries and is ready for inclusions of different genomes, even from other species, representing a reference framework for similar approaches in other genomics contexts.

\section{Construction and content}

\section{Platform architecture}

We implemented NexGenEx - as a role based platform which enables the exploration of NGS based transcriptome collections. The platform was designed to provide enhanced tools for straightforward genome-wide gene expression analyses. As it is shown in Figure 1, the platform is implemented in a Three-tier Architecture schema: 1) Data Tier: Database Management System (DBMS) and Physical Storage Files; 2) Logic Tier: classes and modules deployed on the webserver, and 3) Presentation Tier: a Graphical User Interface (GUI) accessible to the endusers. Figure 1 also describes the main data processing pipelines necessary to define the data to be included in the platform and the resource collections from tomato here included.

The platform works as a web based application running on the .Net Framework 4.0, querying embedded databases, designed and organized in a relational model and implemented in $M y S Q L$, version 5.6.14 InnoDB engine [38]. All key fields and query dependent tuples were 


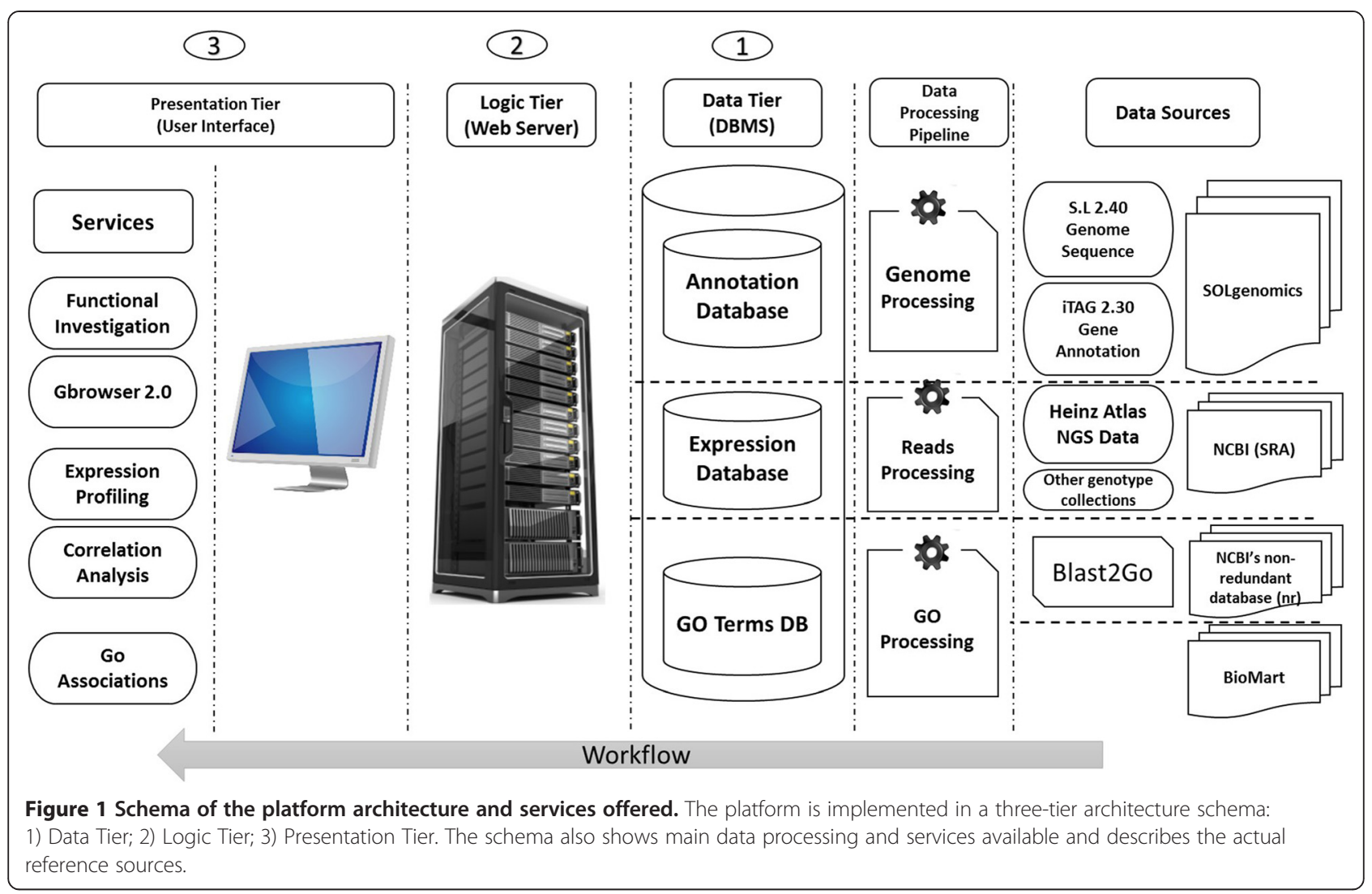

indexed using the BTree indexing algorithm [39]. A Gbrowse [19] database and associated interface are also embedded in the platform.

\section{Data content and processing}

The 12 pseudomolecules sequences representing the version 2.40 of the reference tomato genome, S. lycopersicum cv Heinz, together with the chromosome 0, which includes all the contig sequences not assigned to any other chromosome yet, and the corresponding gene annotation version 2.30 from the international Tomato Annotation Group (iTAG) [30], were downloaded from the Solgenomics website [36]. The genome sequences and the $i T A G$ gene annotation were uploaded in the platform (Figure 1).

The Illumina HiSeq 2000 [40] RNA-seq data collection of Solanum lycopersicum cv Heinz [30] was downloaded from the NCBI SRA archive [SRA: SRP010775]. This collection includes 20 libraries, each one representing one of two biological replicates from 10 different tissues and stages in physiological conditions.

The Illumina HiSeq 2000 RNA-seq data collections from physiological conditions of Solanum lycopersicum cv Ailsa Craig [SRA: SRX098400], including 20 libraries [34], and of Solanum pimpinellifolium [SRA: SRP010775], including 8 libraries [30], were also downloaded from NCBI SRA archive [3].

\section{Illumina reads processing}

Raw Illumina reads were cleaned from adaptor sequences and those with a quality lower than Q20 were discarded using trim galore [41]. Reads shorter than 20 bp were also discarded. Filtered and cleaned reads were indexed by bowtie2 [9] and mapped onto the Tomato pseudomolecules using Tophat2 [10], with default parameters (up to 2 mismatches and intron length of 50 to $500,000 \mathrm{nt}$ ). Ambiguous matches were filtered out, i.e. reads with multiple matches on the genome were eliminated.

The results of reads to genome mapping were then loaded into the Gbrowse system embedded in the platform.

\section{Gene expression analysis}

The gene expression levels for the 34,727 tomato predicted mRNA loci, were defined by the htseq-count software [42], considering reads per each exon. Raw data counting and several normalization methods were considered. The normalized number of reads by the size factor normalization of each tissue/stage in comparison with the size factor of other tissues/stages and the Median (med) normalization technique implemented in the DESeq package [11] were 
also performed; the Reads Per Kilobases Per Million (RPKM) [27] normalization was also independently applied. The expression level results calculated from all mentioned normalization methods together with raw reads counting were uploaded in the platform.

\section{GO term to gene association}

Gene to GO Terms associations were defined by combining two major reference collections: i) the GO reference collection of S. lycopersicum downloaded from the BioMart database [43] (release June 2014), ii) the results of the $S$. lycopersicum mRNA sequences Blast2Go [26] versus the NCBI non-redundant database (nr). The two datasets were then combined removing duplicated terms. The GO annotations associated to the $i$ TAG genes were uploaded into the devoted section of the platform (Figure 1).

\section{Utility}

\section{User interface and database access}

In Figure 2, we report the query page of the NexGenExplatform. The figure shows the main sections users are provided with when consulting the platform content. In the $S$. lycopersicum cv Heinz dedicated partition (which is accessible selecting genome "Tomato", reference "S. lycopersicum Version 2.40") the three available gene expression collections (Heinz, Ailsa Craig, S. pimpinellifolium) can be selected in the collection field. Crosslinks to reference raw collections and to the papers presenting them are also provided.

\section{Results view}

The NexGenEx-Tom platform enables users to investigate expression of the reference tomato genes in different tissues and developmental stages from different collections in physiological conditions. Users can exploit the platform to investigate on a specific gene, or a set of genes. The query can be based on a list of Gene Identifiers (IDs) in the form of Solyc identifiers (e.g. Solyc01g00500), or by indicating one or more functional keywords, or by specifying the boundaries of a chromosome region (indicating the specific directionality of transcription by selecting the strand option). Complex queries can be defined as indicated in the "info" links (Figure 2).

The web-based list of results is organized in an accordion view in which each result set can be investigated in its corresponding section/tab.

In Figure 3, an example query including a list of 5 gene IDs and the corresponding result views are shown.

\section{Annotation of the structure and functional annotation}

By running the query in the system (Figure 3A), the list of the resulting loci associated to the query, including their functional annotation and accessory information from the current gene annotation is reported (Figure 3F).

\section{Expression matrix and profiling}

The expression levels of each queried gene, based on the pre-settings of the query option provided by the user, can be investigated by the selected libraries, in the form of read counts per each locus, median normalized counts or RPKM. As an optional parameter, the average expression level of the replicates from each library can be investigated (Replicate view set to Average, in the query options). In the specific case of the Heinz library two replicates are included in the experiment (Figure 3C).

In addition, a complementary set of statistics, such as the minimum, maximum, average, standard deviation and maximum fold change of the expression levels of each locus in the selected libraries are provided. Moreover, the number of times each of the expression values exceeds the boundaries of one standard deviation from the average is also shown. This value permits to efficiently investigate locus specificities [44]. This section provides a general abstract of the loci expression level behavior in the selected libraries. Moreover, by selecting only 2 different tissues for specific gene sets, bi-comparison of the gene expression fold change are delivered permitting to identify the differential expression levels.

The expression matrix can also be downloaded in CSV format

For each resulting gene, a button (further investigate) has been implemented which enables users to further investigate its gene expression in different conditions (Figure 3C). Indeed, by clicking on this link, the expression level of the corresponding gene will be reported for all the available NGS collections in the platform (Figure 3I), in all the available normalized forms, as calculated for each collection associated to the genome reference. This enables users to focus on the locus of interest with a complete overview of its behavior in any possible and available library per collection.

\section{Expression profiling plot}

The Expression Profiling plot (Figure 3E) shows gene expression variability in different samples from the collection under investigation. This view depends on the number of libraries selected. The possibility to perform the analysis on specific collections of genes, selected by keyword or ID or by a genome region, allows the comparison of the expression profiles of several genes in a straightforward way.

\section{Heatmap visualization}

Heatmaps provide a suitable view on gene expression levels. Customizable heatmaps are offered in the platform to highlight high- and low- expressed genes. This graphical approach is exploited in the platform to show the expression levels of one or multiple genes in different conditions. The data can be reported in the form of 


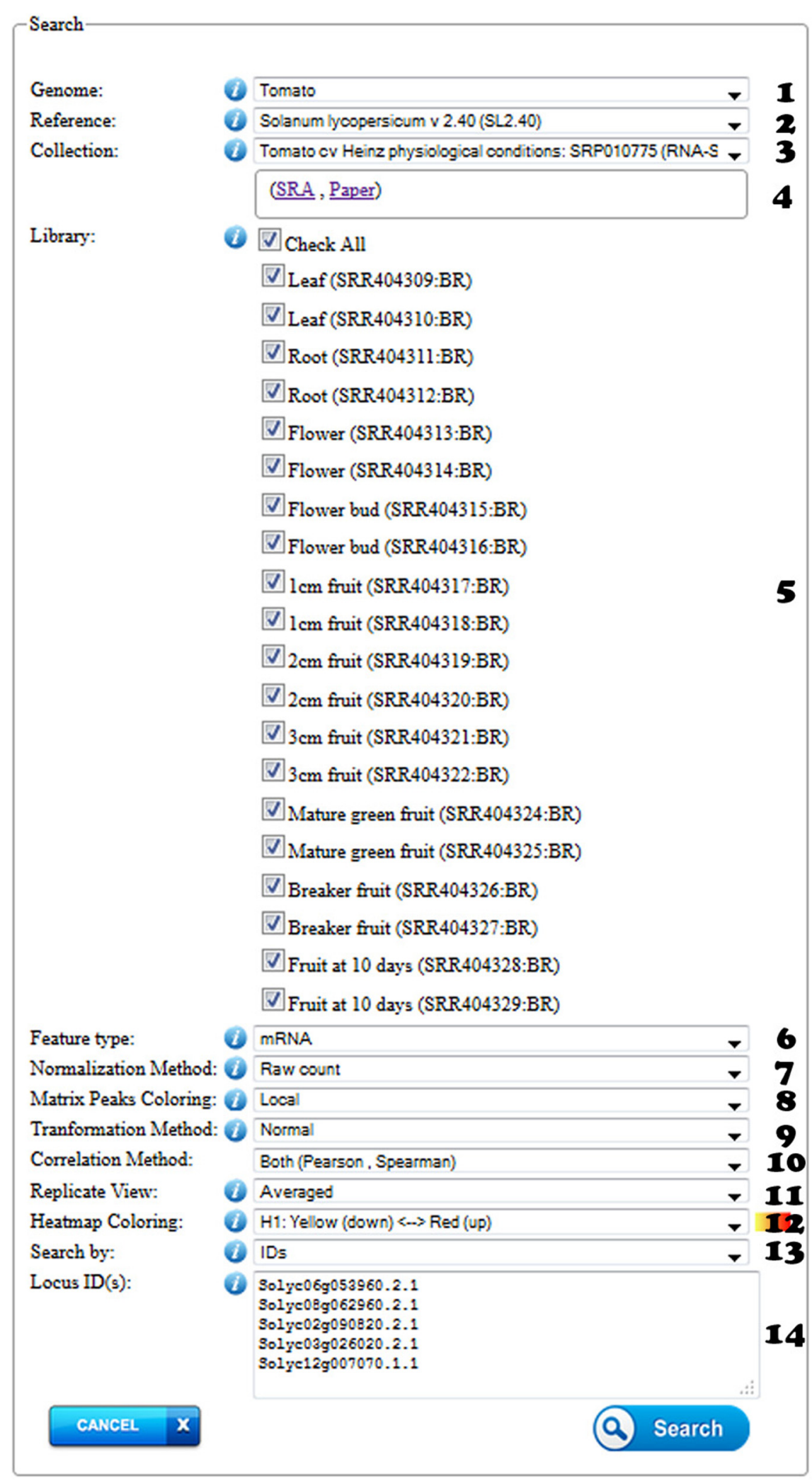

Figure 2 (See legend on next page.) 
(See figure on previous page.)

Figure 2 Main sections provided in the NexGenEx- platform query form. 1) Genome: the genome of interest, e.g. Tomato, 2) Reference: it indicates the reference genotype or cultivar sequences of interest, e.g. Solanum lycopersicum cv Heinz, version 2.40; 3) NGS collections available in the platform, e.g. the Heinz Illumina based RNA-seq collection in physiological condition; 4) link to the data source and paper for this collection; 5) available libraries (replicates/stages/tissues) included in the collection; 6) Feature type: represents the reference genome feature selected for read counting (e.g. mRNA, represents the exons in the locus); 7) Normalization method; 8) Matrix peak coloring, which defines the approach for color coding of the expression levels. This option assigns color frequencies to the cells of a heatmap view comparatively with the expression levels within the query result set (local) or within the whole selected libraries (global). 9) Transformation method: expression levels or their $\log 2$ transformed results can be accessed; 10) Correlation method: Pearson product-moment correlation coefficient or Spareman's correlation coefficient or Both; 11) Replicate view: defines the expression level by each libraries (Separate) or averaged between identical replicates (Average); 12) Heatmap coloring: different heatmap coloring combinations are provided for expression level visualization; 13) Search in: searchable fields can be one/ multiple locus ids (IDs), or simple/multiple functional keywords with advanced selection options (Keyword), or genome regions (Region), and 14) the search area (Locus IDs/Functional keyword/Region): is the text area in which IDs or functional keywords may be listed, or a specific region of the genome may be specified. Accepted formats are described in the information pop-up from the website interface. "Info" buttons are available to support the users.

a matrix, where the level of expression can be marked by a specific color scaling, which may help to highlight high, medium and low levels of expression. The heatmap provided in the NexGenEx- platform (Figure 3C) can be defined by a local or a global scaling, according to the preferred selection in the Matrix Peak Coloring option (Figure 2.8). The "local" heatmap option provides the expression level coloring ranging from the lowest to the highest expression levels resulting from the query. This facilitates the comparison of the specific gene expression levels in the selected set. The "global" coloring option defines the coloring range on the basis of the lowest and highest expression levels in the whole libraries selected during the query. This enables users to identify the gene expression level when compared with the whole expression levels from all the genes in the selected library/ies.

\section{Correlation matrix}

The Correlation matrix analysis illustrates the correlation between genes on the basis of the selected libraries (Figure 3D). The analysis can be based on the Pearson product-moment correlation coefficient, or on the Spearman's rank correlation coefficient, or both at the same time; and the resulting values fluctuates between -1 to 1 , providing the negatively or positively correlated genes. The correlation matrix can be also downloaded in csv format.

\section{GO terms summary table and their association}

As it is shown in Figure 3, a GO Term summary table and the gene to GO Term association to the queried genes is provided to the end users. Figure $3 G$ shows an example of a resulting GO Term summary table of the list of occurring GO Terms, type of GO (in terms of CC: Cellular component, MF: Molecular Function and BP: Biological Process), GO location and the specific GO descriptions. In addition, the enrichment of the GO in the resulting gene set is sorted by $\mathrm{p}$-value for further investigation purposes.
Moreover, the genes to GO Terms association table (Figure $3 \mathrm{H}$ ) also provides the association list of the resulting genes with their GO Terms and their complete description. To further investigate the GO Terms, each $\mathrm{GO}$ is also linked to the AmiGO ontology and annotation database [45].

\section{Genome browser crosslink}

NexGenEx-Tom is enriched with an embedded, customized and updated genome browser interface [19]. The genome browser used, Gbrowse, permits a genome based investigation of the structure of the gene loci included in the database and can be accessed by the selection of each locus from the query set (Figure 4).

Expression profile of the reads mapped on the genome for each tissue/stages is provided in the form of read tracks and coverage plot (xyplot). Another track defined on the basis of all the reads from the available libraries (combined) is also added to provide a general overview of the locus expression for each collection.

Figure 4 shows an example of a Gbrowse based view offered by the platform. This view enables users with indepth investigation of the selected loci and their associated pattern of expression in the form of reads distribution along the genome sequence. The iTAG 2.30 gene annotation is also accessible through the Gbrowse partition. Specifically, the NexGenEx-Tom Gbrowse partition is also enriched with all the annotation tracks included in the ISOL@ platform [46].

\section{A general overview on gene expression analysis from the Heinz genotype}

An example of useful evidence the platform can provide is here reported, considering the Heinz genotype RNAseq collection [30].

Our data analysis highlighted that among all 34727 iTAG annotated genes, 6412 genes show zero read mapped on the transcribed region of the gene locus when considering any of the libraries from each of the 


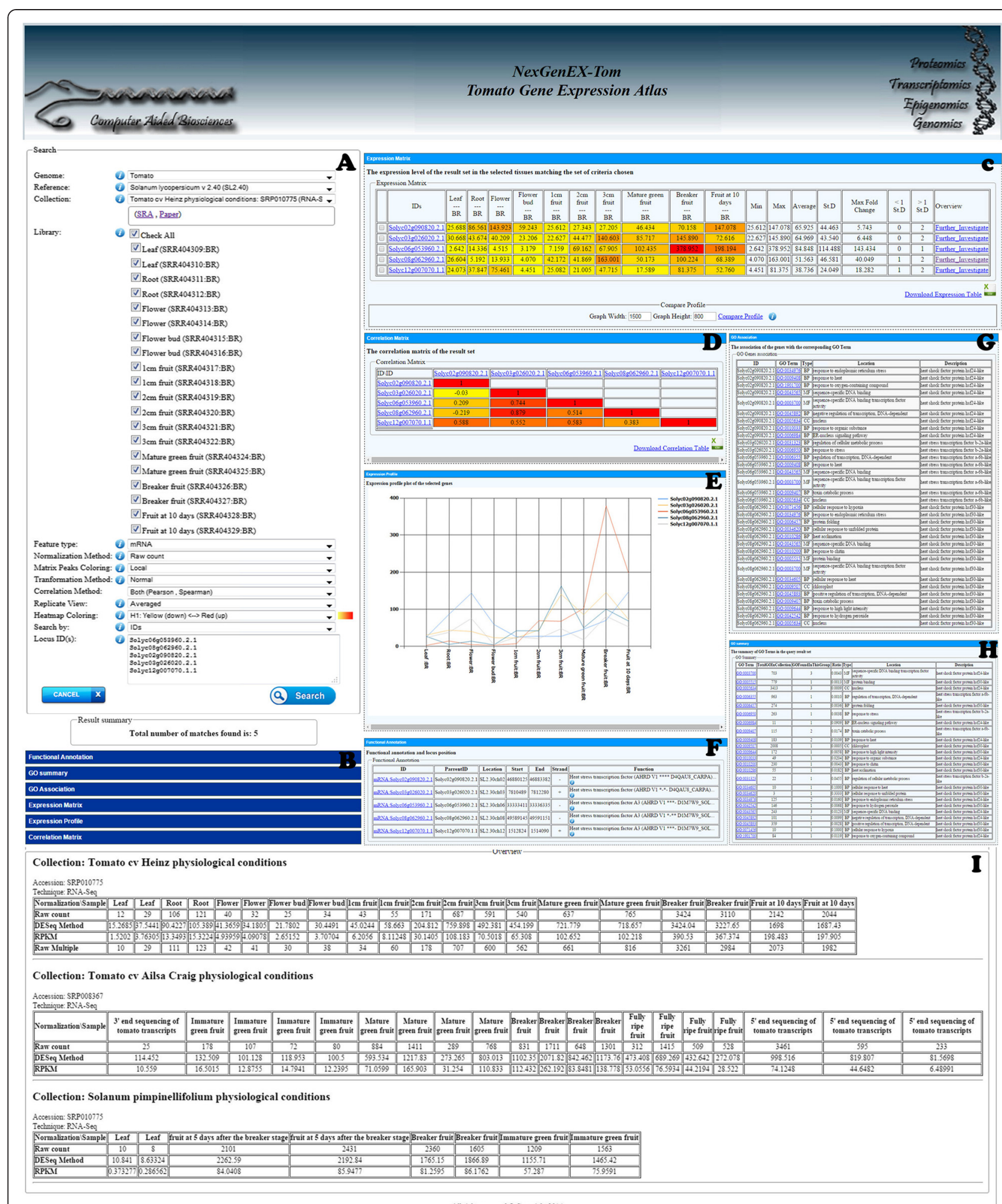

All rights reserved $@$ Copyright 2014

Figure 3 Snapshots of results provided by the platform: A) example query; B) accordion view of all results; C) expression matrix and profiling; D) correlation matrix; E) expression profiling plot; F) functional annotation and locus position on the genome; G) GO Terms summary table; H) GO Terms association table and I) Locus overview from all collections. 


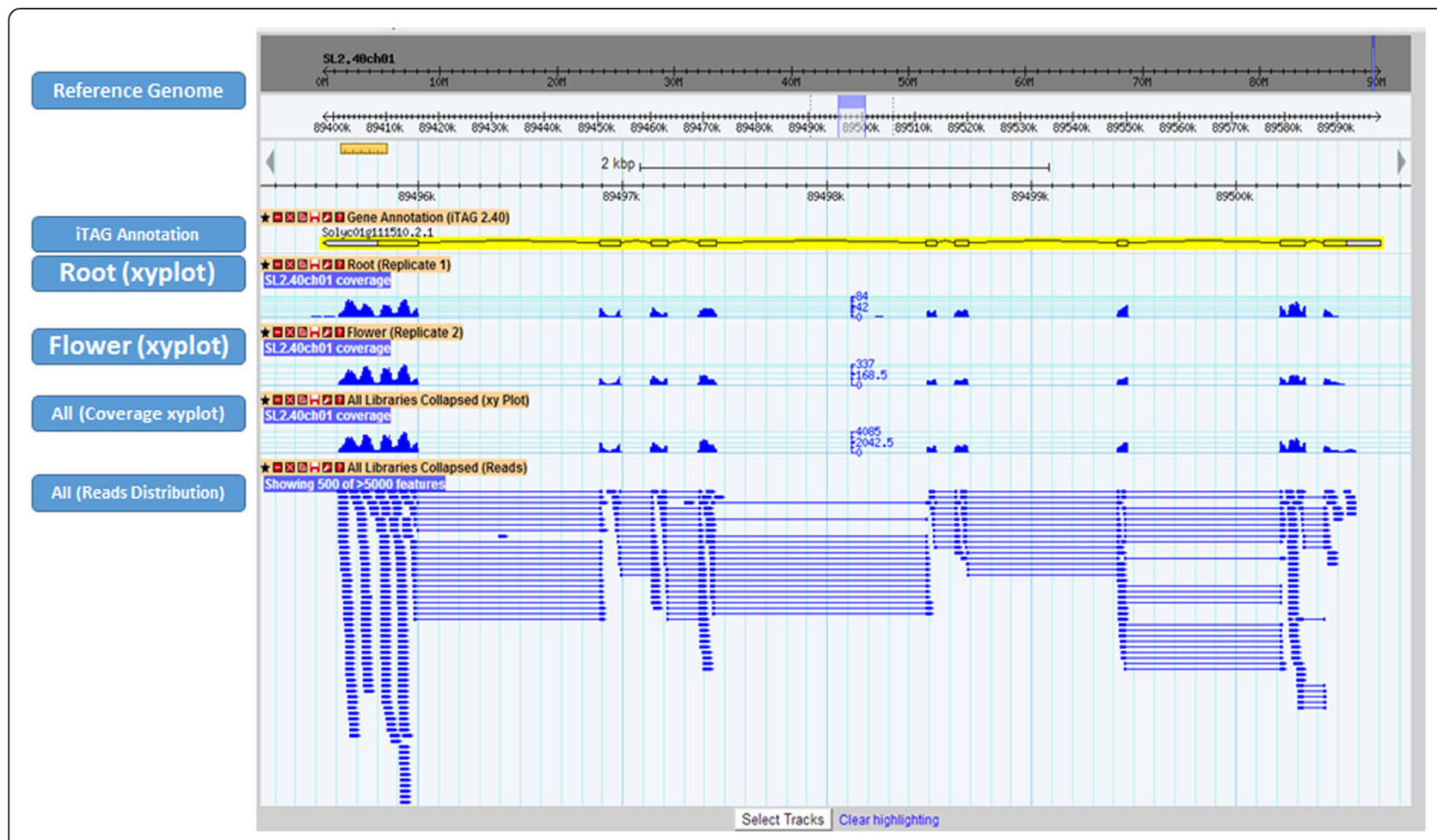

Figure 4 Example of a Gbrowse based view. The gene locus, the xyplot coverage of the NGS reads and their mapping along the selected locus are shown.

replicates of the presented Heinz atlas collection. Interestingly, the number of genes having no read mapped on their gene locus (zero expression level) in the paper of the tomato genome first release [30] is 5700. This is probably due to analytical approaches and highlights the importance of clear description of the methods used to reproduce the data. Moreover, 10025 genes show expression levels lower than 1 RPKM in all the tissues/stages, falling in the criteria to be defined as not expressed genes. Hence, in total, 24702 genes show expression level higher than 1 RPKM in at least one of the investigated physiological conditions. We also analyzed the number of genes that showed the expression level higher than specified thresholds ( 0.3 and 1 RPKM, respectively) only in one condition (defined as specifically expressed). On the other hand, we also reported the list of genes with expression level lower than the given thresholds only in a specific condition (defined as specifically not expressed). In Figure 5, we report the number of specifically expressed and specifically not expressed genes per conditions according to the different thresholds. The statistics shows that comparatively a large number of genes (1106) are specifically expressed in root while a significant number of genes (695) are tissue specifically not expressed in "fruit after 10 days". This overview represents just a limited view of the power of this kind of platforms, including homo-specific and comparable collections.

\section{Discussion}

Though gene expression of tomato tissues has been widely investigated in the years, thanks to both EST sequencing and different microarray platforms, the resulting collections are heterogeneous in terms of experimental approaches employed, genotypes investigated and conditions considered. This makes the reconciliation of the data hard and far from providing a gene expression atlas of the species. The recent release of NGS collections including data from several tissues and stages from physiological conditions for specific genotypes [30,34] contributed a relevant resource to be appropriately exploited for comparative analyses of gene expression patterns in tomato. This paves the way to solve key questions for this species, which is also considered a model for fruit ripening and development. Therefore, an exhaustive organization of the results from the processed collections in a web accessible computational environment, enriched with tools and methods for their explorations, still represents a need for the scientific community.

Many efforts have been undertaken to support the processing and the exploration of results from NGS based collections. Some approaches are mainly devoted 


\begin{tabular}{|c|c|c|c|c|c|c|c|c|}
\hline \multirow[b]{2}{*}{ Library \Threshold } & \multicolumn{4}{|c|}{ Specifically expressed } & \multicolumn{4}{|c|}{ Specifically not expressed } \\
\hline & & 0.3 & & 1 & & 0.3 & & 1 \\
\hline Leaf & [] & 99 & $\square$ & 138 & $\square$ & 158 & $\square$ & 158 \\
\hline Root & $\square$ & 836 & & 1106 & 70 & 123 & $\square$ & 160 \\
\hline Flower & ] & 120 & $\square$ & 105 & I & 44 & ] & 46 \\
\hline Flower bud & - & 624 & 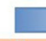 & 630 & I & 45 & $\rrbracket$ & 33 \\
\hline $1 \mathrm{~cm}$ fruit & $\rrbracket$ & 57 & [] & 65 & 】 & 48 & 】 & 62 \\
\hline $2 \mathrm{~cm}$ fruit & $\square$ & 72 & [ & 53 & 1 & 18 & 1 & 13 \\
\hline $3 \mathrm{~cm}$ fruit & $\rrbracket$ & 55 & 【 & 48 & I & 38 & प] & 37 \\
\hline Mature green fruit & $\rrbracket$ & 51 & प & 51 & I & 28 & I & 8 \\
\hline Breaker fruit & I & 26 & | & 19 & $\square$ & 179 & $\square$ & 144 \\
\hline Fruit at 10 days & 凹 & 71 & [] & 64 & 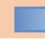 & 756 & & 695 \\
\hline All Fruit & & 9 & & 21 & & 190 & & 235 \\
\hline
\end{tabular}

Figure $\mathbf{5}$ Tissue specific genes in Heinz reference collection. The number of genes with specific behavior is reported considering two different thresholds.

to data visualization, as in the case of genome browser interfaces $[22,18]$. Other efforts result in platforms designed to face the complexity of bioinformatics analyses in genomics, offering free [13-15] or under payment tools $[16,17]$.

The platforms may allow the setup of customizable workflows $[13,14]$, or are based on pre-defined pipelines [15], providing services and methods for results investigation and reproducibility. However, though based on standards, the data processing requires, in most cases, user's awareness on methods and parameters, as well as a constant updating on data requirements and novel tools, due to the fast evolution in the field.

While choosing the correct framework to tune the analyses and selecting the reliable resources to integrate in each specific context can be a challenge for the endusers, several platforms have been proposed to overcome these issues. Some efforts, therefore, such as RNASeqExpressionBrowser [20], mainly aimed to contribute to data integration and exploration thanks to suitable web interfaces for querying the RNA-seq expression results, that can be easily loaded as long as they are represented in a matrix-like format. In alternative, web based resources have been developed to offer immediate views on the results. For instance, in plants, several databases organizes NGS based collections [for the TIGR, soybean, Maize, TGRD, SGN and TFGD]. These platforms, each in a specific context, usually are devoted to organize the main NGS data for a species and, often, also analytical tools and appropriate query systems, possibly including other added-value resources in the specific genomics context.

Gene expression data from several technological approaches (ESTs, microarray, RNA-seq) dedicated to transcriptome analyses have been expanded during years of research in Tomato genomics. TFGD [35] offers a reference site to collect several of the tomato gene expression related collections, also including, per each RNA-seq based collection, tools and query interfaces to access gene lists and their RPKM normalized values per sample. Moreover, dedicated platforms to allow exploration of NGS reads mapped onto genome sequences, permitting the visualization of their distribution along each chromosome, are available at the SGN website [36], ISOL@ [46], TGDR [37], SPUD [25].

However, suitably organized processed collections to offer enhanced and flexible NGS based analyses for tomato are still required. In this context, we implemented NexGenEx-. We aimed to offer processed collections, providing a user-friendly platform and tools for exploitation of gene expression analysis based on a genome reference and, possibly, on accessory information available from the species.

NexGenEx-Tom is the tomato dedicated partition of the NexGenEx- platform, specifically designed to allow investigations on NGS based transcriptome collections representative of different tissues/stages from different genotypes [30,34].

It offers a novel resource that permits to explore gene expression by library, by genotype, and comparatively between genotypes, highlighting possible peculiarities and specificities. This effort is also relevant as a reference at the light of the manifold similar experiments that are being undertaken in tomato.

The flexibility and the customizable bioinformatics supported by NexGenEx- enables different views and representations of the embedded results. The access to cleaned and processed data, differently normalized collections (such as the median normalized, not considering the length bias, and the RPKM, including the effect of the length bias), the rescaling based on the $\log 2$ transformation, the possibility to select one or multiple genes, from part or all libraries, considering or not the replicate contribution, provide a novel bioinformatics bench-work for immediate investigations and a user-friendly computational environment.

The statistical report section, including minimum, maximum, average, standard deviation and the maximum fold 
change of the expression levels in the selected libraries, also provides support to investigate specificities for the desired query set.

The query by Gene Identifier(s), or by Functional Keyword(s) or by specifying the boundaries of a chromosome region, based on an efficient data management system, permits complex conceptual browsing of the platform information content. Associated to the gene list and the corresponding functional annotation, an updated Gene Ontology report is provided.

A useful feature available in NexGenEx- is the possibility to straightforward investigate gene expression levels in different formats from all the libraries of several collections. This permits comparative analyses based on the same genome reference.

NexGenEx- also includes the direct link to a genome browser interface, thanks to the embedded Gbrowse interface [22]. This allows to visualize reads mapped to the genome by library, even when representing different replicates, integrated with the available genome annotation, offering a deep view on read to exon distribution, and, therefore, highlighting possible peculiarities such as alternative exons in a locus. Specifically, the Gbrowse database included in NexGenEx-Tom is also enriched with the data collected from the tomato genome browser implemented in ISOL@ [46], permitting to enrich by several additional tracks the whole resource and further support genome based gene expression analyses.

Beyond providing a user-friendly interface to investigate comprehensive reference collections, the platform was designed with the aim to easily be expanded with other NGS based data. It can also integrate different genome releases, possibly from different cultivars or genotypes, but even from different species. As a consequence, though the platform is proposed as an effort in tomato genomics, it can represent a useful tool also for other similar species, permitting the gene based exploitation of these precious and challenging datasets even by bioinformatics non expert users.

\section{Conclusions}

The emergence of NGS technologies opened an unexpected precious window to a deeper understanding of genome organization and gene expression mechanisms, regulation and control. Their contribution is enhanced by suitable experimental project design, in the form of gene expression atlases that could highlight the coordination of the events that make the genome express its genetic information in physiological or alternative conditions. This strongly requires the corresponding organization of bioinformatics environments where the results from processed data are appropriately organized and may be straightforward investigated and compared even by users with limited experience in bioinformatics. NexGenEx- aims to give a contribution to this challenge and here we described its main peculiarities considering the partition NexGenEx-Tom, organizing tomato gene expression collections based on Illumina data.

Solanum lycopersicum cv Heinz has been selected to represent the reference tomato genome and also as a reference to support comparative analysis within the Solanaceae family. The availability of related consistent transcriptome collections, can further highlight the main functionality of this genome representing a profitable resource for all the interested scientific community.

In the presented version, NexGenEx-Tom aims to contribute to gene expression analyses in tomato offering three relevant processed NGS based transcriptome collections and user-friendly tools to permit their exploration.

Though our main purpose at this stage was to provide a bioinformatics environment for supporting genome wide investigations based on the reference tomato genome in physiological conditions, the platform architecture has been designed and is proposed as a model approach for similar efforts. Moreover, at the light of the rapid expansion of NGS based efforts, the platform is already organized to be expanded with other transcriptome collections, possibly from different genotypes, and eventually even from different species, representing a model strategy to face the challenge and the opportunity offered by these precious data resources.

\section{Availability and requirements}

Access to NexGenEx-Tom is freely available for research purposes, for users from non-profit and academic organizations at http://www.cab.unina.it/NexGenEx-Tom.

The platform is designed in a role based architecture which also enables dedicated access to private data after authentication (password protected section) for collaborative efforts and to protect still unpublished results upon user specific requests. Interested users are invited to follow the contacts for agreements on the inclusions of their collections in the platforms as collaborative efforts or as a service.

The platform is also supported with specific Application Program Interfaces (APIs) to enable, upon request, interested developers to deploy in-house queries within their system, getting advantage of available data and analysis in the NexGenEx- system.

\section{Competing interests}

The authors declare that they have no competing interests.

\section{Authors' contributions}

$\mathrm{HB}$ initiated the idea of the NexGenEx- platform, designed and implemented the system and carried out the data analysis. MLC proposed the project and supervised the work. Both authors wrote, read and approved the final manuscript. 


\section{Acknowledgements}

Hamed Bostan is a PhD fellow supported by the Solanaceae Pollen Thermotolerance - Marie Curie Initial Training Network (SPOT-ITN) project (Grant agreement 289220).

This work is also supported by the Genopom PRO Projects (Ministero dell'Istruzione, dell'Università e della Ricerca (MIUR), Italy) and is in the frame of the Cost Action FA1106.

We acknowledge Dr. Guido Incerti for his support, Dr. Valentino Ruggieri and the anonymous reviewers for their useful suggestions to improve the manuscript.

Received: 1 July 2014 Accepted: 30 December 2014

Published online: 13 February 2015

\section{References}

1. Jimenez-Lopez JC, Gachomo EW, Sharma S, Kotchoni SO. Genome sequencing and next-generation sequence data analysis: A comprehensive compilation of bioinformatics tools and databases. Am J Mol Biol. 2013;3:115.

2. Metzker ML. Sequencing technologies-the next generation. Nat Rev Genet. 2009;11:31-46.

3. Leinonen R, Sugawara H, Shumway M. The sequence read archive. Nucleic Acids Res. 2011;39:D19-21.

4. Barrett T, Troup DB, Wilhite SE, Ledoux P, Rudnev D, Evangelista C, et al. NCBI GEO: mining tens of millions of expression profiles-database and tools update. Nucleic Acids Res. 2007:35:D760-5.

5. Martin M. Cutadapt removes adapter sequences from high-throughput sequencing reads. EMBnet journal. 2011;17:10-2.

6. Andrews S: FastQC: A quality control tool for high throughput sequence data. http://www.bioinformatics.babraham.ac.uk/projects/fastqc/ 2010

7. Grabherr MG, Haas BJ, Yassour M, Levin JZ, Thompson DA, Amit I, et al. Full-length transcriptome assembly from RNA-Seq data without a reference genome. Nat Biotechnol. 2011;29:644-52.

8. Schulz MH, Zerbino DR, Vingron M, Birney E. Oases: robust de novo RNA-seq assembly across the dynamic range of expression levels. Bioinformatics. 2012;28:1086-92.

9. $\quad$ Langmead B, Salzberg SL. Fast gapped-read alignment with Bowtie 2 . Nat Methods. 2012;9:357-9.

10. Kim D, Pertea G, Trapnell C, Pimentel H, Kelley R, Salzberg SL. TopHat2: accurate alignment of transcriptomes in the presence of insertions, deletions and gene fusions. Genome Biol. 2013;14:R36.

11. Anders S: Analysing RNA-Seq data with the DESeq package. Molecular biology 2010:1-17

12. Anders S, McCarthy DJ, Chen Y, Okoniewski M, Smyth GK, Huber W, et al. Count-based differential expression analysis of RNA sequencing data using $R$ and Bioconductor. Nat Protoc. 2013:8:1765-86.

13. Giardine B, Riemer C, Hardison RC, Burhans R, Elnitski L, Shah P, et al. Galaxy: a platform for interactive large-scale genome analysis. Genome Res. 2005;15:1451-5.

14. Reich M, Liefeld T, Gould J, Lerner J, Tamayo P, Mesirov JP. GenePattern 2.0. Nat Genet. 2006;38:500-1

15. Boria I, Pesole G, Mignone F. NGS TREX: next generation sequences transcriptome profile explorer. EMBnet journal. 2012;18:93.

16. Coletta A, Molter C, Duqué R, Steenhoff D, Taminau J, De Schaetzen V, et al. InSilico DB genomic datasets hub: an efficient starting point for analyzing genome-wide studies in GenePattern, Integrative Genomics Viewer, and R/Bioconductor. Genome Biol. 2012;13:R104.

17. Forsberg R, Mønsted S, Hein AM: CLC Bio Integrated Platform for Handling and Analysis of Tag Sequencing Data. Tag-Based Next Generation Sequencing (eds M Harbers and G Kahl), Wiley-VCH Verlag GmbH \& Co KGaA, Weinheim, Germany 2011:393-405.

18. Thorvaldsdóttir H, Robinson JT, Mesirov JP. Integrative Genomics Viewer (IGV): high-performance genomics data visualization and exploration. Brief Bioinform. 2013;14(2):178-92.

19. Stein LD, Mungall C, Shu S, Caudy M, Mangone M, Day A, et al. The generic genome browser: a building block for a model organism system database. Genome Res. 2002;12:1599-610.

20. Nussbaumer T, Kugler KG, Bader KC, Sharma S, Seidel M, Mayer KF. RNASegExpressionBrowser-A web interface to browse and visualize high-throughput expression data. Bioinformatics. 2014;30:2519-20.
21. Fernández XM, Birney E: Ensembl Genome Browser. In Vogel and Motulsky's Human Genetics. Springer Berlin Heidelberg; 2010: 923-939

22. Kent WJ, Sugnet CW, Furey TS, Roskin KM, Pringle TH, Zahler AM, et al. The human genome browser at UCSC. Genome Res. 2002;12:996-1006.

23. Ouyang S, Zhu W, Hamilton J, Lin H, Campbell M, Childs K, et al. The TIGR rice genome annotation resource: improvements and new features. Nucleic Acids Res. 2007;35:D883-7.

24. Grant D, Nelson RT, Cannon SB, Shoemaker RC. SoyBase, the USDA-ARS soybean genetics and genomics database. Nucleic Acids Res. 2009;38:D843-846.

25. Hirsch CD, Hamilton JP, Childs KL, Cepela J, Crisovan E, Vaillancourt B, Hirsch CN, Habermann M, Neal B, Buell CR: Spud DB: A resource for mining sequences, genotypes, and phenotypes to accelerate potato breeding. The Plant Genome 2014, 7

26. Trapnell C, Roberts A, Goff L, Pertea G, Kim D, Kelley DR, et al. Differential gene and transcript expression analysis of RNA-seq experiments with TopHat and Cufflinks. Nat Protoc. 2012:7:562-78.

27. Mortazavi A, Williams BA, McCue K, Schaeffer L, Wold B. Mapping and quantifying mammalian transcriptomes by RNA-Seq. Nat Methods. 2008:5:621-8.

28. Sekhon RS, Briskine R, Hirsch CN, Myers CL, Springer NM, Buell CR, et al. Maize gene atlas developed by RNA sequencing and comparative evaluation of transcriptomes based on RNA sequencing and microarrays. PLoS One. 2013;8:e61005

29. Jamie A, Iniguez LP, Fu F, Bucciarelli B, Miller SS, Jackson SA, et al. An RNA-Seq based gene expression atlas of the common bean. BMC Genomics. 2014;15:866.

30. Consortium TG. The tomato genome sequence provides insights into fleshy fruit evolution. Nature. 2012:485:635-41.

31. Consortium PGS. Genome sequence and analysis of the tuber crop potato. Nature. 2011;475:189-95.

32. Kim S, Park M, Yeom S-I, Kim Y-M, Lee JM, Lee H-A, et al. Genome sequence of the hot pepper provides insights into the evolution of pungency in Capsicum species. Nat Genet. 2014:46:270-8.

33. Hirakawa H, Shirasawa K, Miyatake K, Nunome T, Negoro S, Ohyama A, Yamaguchi H, Sato S, Isobe S, Tabata S, Fukuoka H: Draft Genome Sequence of Eggplant (Solanum melongena L.): the Representative Solanum Species Indigenous to the Old World. DNA Research 2014:1-12.

34. Zhong S, Fei Z, Chen Y-R, Zheng Y, Huang M, Vrebalov J, et al. Single-base resolution methylomes of tomato fruit development reveal epigenome modifications associated with ripening. Nat Biotechnol. 2013;31:154-9.

35. Fei $Z$, Joung J-G, Tang $X$, Zheng Y, Huang M, Lee JM, et al. Tomato Functional Genomics Database: a comprehensive resource and analysis package for tomato functional genomics. Nucleic Acids Res. 2011:39:D1156-63.

36. Mueller LA, Solow TH, Taylor N, Skwarecki B, Buels R, Binns J, et al. The SOL Genomics Network. A comparative resource for Solanaceae biology and beyond. Plant Physiol. 2005;138:1310-7.

37. Suresh BV, Roy R, Sahu K, Misra G, Chattopadhyay D. Tomato Genomic Resources Database: An Integrated Repository of Useful Tomato Genomic Information for Basic and Applied Research. PLoS One. 2014:9:e86387.

38. Fruhwirt P, Huber M, Mulazzani M, Weippl ER. Innodb database forensics. In: Advanced Information Networking and Applications (AINA), 2010 24th IEEE International Conference on; 20-23 April 2010. Perth, Western Australia: IEEE; 2010. p. 1028-36.

39. Bayer R: The universal B-tree for multidimensional indexing: General concepts. In. Mathematisches Institut und Institut für Informatik der Technischen Universität München: Springer; 1997: 198-209

40. Caporaso JG, Lauber CL, Walters WA, Berg-Lyons D, Huntley J, Fierer N, et al. Ultra-high-throughput microbial community analysis on the Illumina HiSeq and MiSeq platforms. The ISME journal. 2012;6:1621-4.

41. Trim Galore [http://www.bioinformatics.babraham.ac.uk/projects/ trim_galore/]

42. A Python framework to work with high-throughput sequencing data, Bioinformatics, in print, online at doi:10.1093/bioinformatics/btu638 2014

43. Kinsella RJ, Kähäri A, Haider S, Zamora J, Proctor G, Spudich G, et al. Ensembl BioMarts: a hub for data retrieval across taxonomic space. Database. 2011:2011:bar030.

44. Seo S: A review and comparison of methods for detecting outliers in univariate data sets. University of Pittsburgh, Pennsylvania, Graduate School of Public Health, Biostatistics; 2006. 
45. Carbon S, Ireland A, Mungall CJ, Shu S, Marshall B, Lewis S. AmiGO: online access to ontology and annotation data. Bioinformatics. 2009;25:288-9.

46. Chiusano ML, D'Agostino N, Traini A, Licciardello C, Raimondo E, Aversano

M, et al. ISOL@: an Italian SOLAnaceae genomics resource. BMC

Bioinformatics. 2008;9:57.

Submit your next manuscript to BioMed Central and take full advantage of:

- Convenient online submission

- Thorough peer review

- No space constraints or color figure charges

- Immediate publication on acceptance

- Inclusion in PubMed, CAS, Scopus and Google Scholar

- Research which is freely available for redistribution 